\title{
Frequency of firefighters' heat-related illness and its association with removing personal protective equipment and working hours
}

\author{
Siyeon KIM${ }^{1,2}$, Do-Hee KIM ${ }^{3}$, Hyo-Hyun $\operatorname{LEE}^{3}$ and Joo-Young $\operatorname{LEE}^{1,3 *}$ \\ ${ }^{1}$ Department of Textiles, Merchandising and Fashion Design, Seoul National University, Republic of Korea \\ ${ }^{2}$ Human Convergence Technology R\&D Group, Korea Institute of Industrial Technology, Republic of Korea \\ ${ }^{3}$ Research Institute of Human Ecology, Seoul National University, Republic of Korea
}

Received March 16, 2018 and accepted September 3, 2018

Published online in J-STAGE September 11, 2018

\begin{abstract}
The purpose of this study was to examine the frequency of firefighters' heat-related illness (HRI) in the line of duty with relation to resting/working hours and the behaviour of taking off PPE during rest periods based on nationwide survey $(\mathrm{N}=674)$. The results showed that $74.8 \%$ of firefighters experienced HRI symptoms and 5\% of firefighters suffered from symptoms of HRI 20 times or more in a year. This study also showed a statistically significant correlation between working hours and removing PPE with the experience of HRI symptoms $(p<0.05)$. In addition, removing PPE except the helmet was correlated with resting hours $(p<0.001$; Gloves: $p=0.051)$, which indicates that removing PPE is related to resting time. This study revealed that firefighters routinely experienced mild HRI symptoms and the fatalities were just a tiny fraction. It is also emphasized that attention should be given on the significant role of taking off PPE during rest and on the standardized firefighters' rehabilitation followed by its application.
\end{abstract}

Key words: Firefighters' heat strain, Heat-related illness, Firefighters' rehabilitation, Personal protective clothing, Occupational health

\section{Introduction}

Heat-related illness (HRI) is a pathological state caused by the elevation of body core temperature. It can be caused by excessive physical activity and high environmental temperature combined with restricted heat dissipation due to clothing ${ }^{1)}$. Milder forms of HRI (e.g., heat exhaustion) related to a core temperature of less than $40^{\circ} \mathrm{C}$ do not damage the central nervous system (CNS), but heat stroke can evoke fatal damage to the CNS unless immediate and

*To whom correspondence should be addressed. E-mail: leex3140@snu.ac.kr

(C)2019 National Institute of Occupational Safety and Health appropriate treatment is given. Core temperature is a reliable criterion to diagnose HRI which can also be noticed by symptoms like headache, dizziness, nausea, vomiting, confusion, and syncope ${ }^{2,3)}$. Due to the difficulty of immediately measuring core temperature without assistance by medical personnel, a rapid response to these symptoms followed by cooling strategies can prevent HRI from progressing to heat stroke, which can cause injury or death.

However, it is not always easy to initiate appropriate and sufficient treatment right after detecting HRI symptoms in the case of firefighters in the fire scenes where a combination of work in dangerous environments where heat and flames, intensive physical activity and encapsulated personal protective equipment (PPE) make cooling strategies challenging ${ }^{4}$. Rectal temperature increases to 
$39.0^{\circ} \mathrm{C}$ within $40 \mathrm{~min}$ in air temperature of $35^{\circ} \mathrm{C}$ with heavy work and full firefighters' $\mathrm{PPE}^{5)}$. Although the limited capacity of self-contained breathing apparatus requires firefighters to come out from fire scenes every 30 $60 \mathrm{~min}^{6}$, they are regularly obliged to re-enter fire scenes during long emergency events until completing fire suppression without sufficient rest between work bouts ${ }^{7}$. HRI can occur without any notice to their actual physiological burden because thermal sensation and perceived exertion is not parallel with physiological heat strain ${ }^{8)}$. In the US, wildland firefighters' fatalities related to heat stroke was 255 in total over a $10 \mathrm{yr}$ period $^{9}$, and the occurrence of HRI in firefighters in Washington State was the second highest among various occupations over a $10 \mathrm{yr} \operatorname{span}^{10)}$. Also, in Korea, one death in the line of duty occurred in 2013 after prolonged firefighting in the summer. However, in comparison with the danger of HRI to firefighters, we know little about the actual prevalence of HRI on firefighters except in cases that ended in death. Even though HRI does not directly cause irreversible injury or death, it can deteriorate their performances and be related to dysfunction and other disease or injuries ${ }^{11-13)}$.

As an attempt to prevent firefighters' HRI, rehabilitation is essential to mitigate the physical and physiological stress of firefighting ${ }^{14)}$. NFPA 1584 states that rest and recovery time should be more than $10 \mathrm{~min}$ after the 1 st use of self-contained breathing apparatus (SCBA) and more than 20 min after the 2nd use of SCBA along with detailed recommendations about rehydration and active cooling during the rest and recovery period ${ }^{15)}$. However, there is no obligation to observe the standard, and the actual procedure varied according to countries and administrative districts. In particular, in South Korea, no standardized rehabilitating approaches exist. According to article 22 of the firefighters' health and safety management regulations in Korea, commanders should inspect firefighters' physical health condition every shift to refrain from continuing their work with health problems ${ }^{16}$. However, there is not any criterion for this quick diagnosis, explanation about duration of rest and work periods, nor any recommendation for the rehabilitating measures. On the other hand, among various ways to cool the body during a rest period, simply taking off PPE can reduce physiological heat strain by promoting evaporative sweat rate ${ }^{5)}$. However, firefighters often face difficulty in donning/doffing their sweatsoaked PPE ${ }^{17)}$ in the short rest period with uncertainty of re-entering fire scenes ${ }^{8}$, which can hinder even passive cooling during the rest period. In addition, a risk of chemical exposure could be increased during doffing PPE by inhalation exposure to off-gassing contaminants and a direct contact to bare skin with contaminated gear ${ }^{18)}$.

As far as we know, this is the first study to examine the frequency of firefighters' HRI in the line of duty with relation to resting/working hours and the behaviour of taking off PPE during rest periods. A nationwide survey with Korean firefighters was conducted in order to quantitatively evaluate the effects of those factors on firefighters' HRI.

\section{Methods}

\section{Sample}

The survey was directed towards Korean professional firefighters who have taken charge of suppressing fire or rescuing before. Firefighters in charge of only medical emergency care or office work were excluded because they are not directly exposed to excessive heat strain at fire scenes. In April 2016, printed copies of a questionnaire were distributed nationwide to 1,050 Korean professional firefighters via Fire \& Disaster Headquarters. The distributed areas included the following administrative districts: Seoul, Geonggi-do, Chungchungnam-do, Junranum-do, and Gangwon-do. Predominant cause and place of the fire accidents are different by district. In Seoul, capital city of Korea, structural firefighting prevails, and wildland firefighting is rarely found. On the other hand, wildland fires are much frequent in the mountainous territory of Gangwon-do. In order to consider this regional distinct of firefighting, respondents were recruited in various regions. Most responses came from Seoul in consideration of the largest number of firefighters in Seoul. Of 1,050 questionnaires, 983 were collected. Among them, responses without demographic information $(\mathrm{N}=177)$ and those answered by firefighters who have never been in charge of suppressing fire or rescuing work $(\mathrm{N}=132)$ were excluded in the analysis. In the end, 674 questionnaires were used in the final analysis, and the demographic and occupational information were presented in (Table 1). Prior to participation of the survey, written consent was obtained from all respondents, and the entire procedure of this study was approved by the Institutional Review Board of Seoul National University (IRB No. E1602/001-005).

\section{Questionnaire construction}

The current questionnaire was a part of an extensive questionnaire which encompassed various issues on firefighters' safety and their personal protective equipment. The series of questions related to firefighters' heat strain were placed in the initial part, and they contained five 
Table 1. Demographic characteristics of the respondents

\begin{tabular}{|c|c|c|}
\hline & No. & $\%$ \\
\hline Total respondents & 674 & 100 \\
\hline \multicolumn{3}{|l|}{ Sex } \\
\hline Male & 657 & 97.5 \\
\hline Female & 14 & 2.1 \\
\hline No details & 3 & 0.4 \\
\hline \multicolumn{3}{|l|}{ Age (yr) } \\
\hline $20-29$ & 71 & 10.5 \\
\hline $30-39$ & 303 & 45.0 \\
\hline $40-49$ & 206 & 30.6 \\
\hline 50 and over & 88 & 12.9 \\
\hline No details & 6 & 0.4 \\
\hline \multicolumn{3}{|l|}{ Duration of each job (yr) } \\
\hline \multicolumn{3}{|l|}{ Fire suppression } \\
\hline$<1$ & 138 & 20.5 \\
\hline $1-4$ & 240 & 35.6 \\
\hline$\geq 5$ & 293 & 43.5 \\
\hline No details ${ }^{\mathrm{a}}$ & 3 & 0.4 \\
\hline \multicolumn{3}{|l|}{ Emergency rescue } \\
\hline$<1$ & 431 & 63.9 \\
\hline $1-4$ & 113 & 16.8 \\
\hline$\geq 5$ & 127 & 18.8 \\
\hline No details ${ }^{\mathrm{a}}$ & 3 & 0.4 \\
\hline \multicolumn{3}{|c|}{ Present place of employment } \\
\hline Seoul & 211 & 31.3 \\
\hline Geonggi-do & 179 & 26.6 \\
\hline Chungchungnam-do & 75 & 11.1 \\
\hline Jeonranam-do & 83 & 12.3 \\
\hline Gangwon-do & 78 & 11.6 \\
\hline Others & 48 & 7.1 \\
\hline
\end{tabular}

aThese answers were included in analysis because they reported the existence of fire suppressing and emergency rescuing experiences even though they did not report the detailed information of duration of each job.

bundles of questions associated with heat-related illness and the environmental and behavioural factors related to heat strain in their work place; experiences of heat-related illness, duration time of rest and work periods, drinking habits during resting and frequencies of wearing of PPE. Also, demographic information included age, sex, possession of the certificate of emergency medical technician, and detailed information about their work periods and specific operations they have taken charge of. Prior to the survey, ten professional firefighters with more than $20 \mathrm{yr}$ in working experience inspected the draft. They advised on the appropriateness of questions and words used in all questions and answers given in multiple choice questions. In a final questionnaire, 27 questions were included with 79 sub-questions (46 multiple choice questions and 33 short-answer questions).

At first, in order to investigate the occurrence of firefighters' HRI, a series of yes/no items regarding symptoms of HRI were elicited. Respondents gave short answers on how frequently they experienced each symptom during a year. Symptoms of heat-related illness contained headache, sudden muscle cramps, dizziness, nausea, vomiting, and fainting ${ }^{3,19)}$. It was also asked whether they reported these symptoms to their authority and the reasons why they did not report it. Regarding the reasons, multiple choices were given as follows: 1) it required little or no medical expenses: 2) people usually do not report it; 3 ) concerns of disadvantage for promotion; 4) it required a complex process; 5) troublesome; 6) it is natural to experience those symptoms at fire scenes. Participants also responded to questions about duration of rest and work period with details about the average, minimum, and maximum values. It was asked separately according to the size of the fire accident (small, medium and conflagration), because there are differences in supplies and the resting place for firefighters in case of conflagration in contrast to small/medium sized fire scenes. Also, time for duration of single work periods and total duration time were inquired separately for working hours, because their working times tend to be divided by limited capacity of SCBA at the fire scenes.

Firefighters also responded about the frequency of taking off PPE during their rest periods; always, usually, hardly, and never taking off PPE at rest. Components of PPE included the helmet, hood, gloves, boots, bunker jacket and pants. SCBA was not included because it is obliged to be taken off for replacement or recharge during rest periods, and removing SCBA is not an option for cooling body during rest. In particular, regarding the bunker jacket, another option is to just open it without totally taking it off. This was additionally considered.

\section{Statistical methods}

Three quartiles with 95th percentiles of data about frequency of heat-related illness and duration time of rest/ work periods were reported to descript data values. In order to investigate the effect of rest and working duration time on the frequency of HRI symptoms, Pearson's correlation test was conducted with log transformation for both of the variables and Pearson correlation coefficients $(r)$ were reported. Odd ratios were calculated to quantify the influence of wearing PPE at rest on presence of HRI symptoms. In this calculation, wearing frequency of PPE was divided into two groups; people who always or usually wear PPE at rest and people who hardly or rarely 
Table 2. Experience frequency of symptoms of heat-related illness (HRI) in firefighters' working place

\begin{tabular}{|c|c|c|c|c|c|c|c|c|c|}
\hline \multirow{3}{*}{ HRI symptoms experienced } & \multirow{3}{*}{$\begin{array}{c}\text { No. of } \\
\text { Respondents }\end{array}$} & \multirow{2}{*}{\multicolumn{2}{|c|}{$\begin{array}{c}\text { Number of } \\
\text { experienced firefighters }\end{array}$}} & \multicolumn{6}{|c|}{ Frequency of experience per year ${ }^{\mathrm{a}}$} \\
\hline & & & & \multicolumn{6}{|c|}{ Percentiles } \\
\hline & & $\mathrm{N}$ & $\%$ & $\mathrm{~N}$ & 25 th & 50 th & 75 th & 95 th & 98th \\
\hline \multicolumn{10}{|l|}{ HRI symptoms } \\
\hline Dizziness & 669 & 379 & 56.6 & 373 & $2(0)$ & $3(1)$ & $10(4)$ & $44(20)$ & $95.2(63.5)$ \\
\hline Headache & 667 & 326 & 48.8 & 320 & $2(0)$ & $4(0)$ & $10(3)$ & $35.95(20)$ & $100(55)$ \\
\hline Nausea & 667 & 317 & 47.5 & 313 & $2(0)$ & $3(0)$ & $10(3)$ & $50(20)$ & $107.2(50)$ \\
\hline Muscle cramps & 665 & 304 & 45.7 & 303 & $1.5(0)$ & $3(0)$ & $10(2)$ & $30(19.25)$ & $50(30)$ \\
\hline Confusion & 665 & 221 & 33.2 & 215 & $2(0)$ & $3(0)$ & $10(1.63)$ & $42(12.25)$ & $66.8(30)$ \\
\hline Vomiting & 661 & 151 & 22.8 & 147 & $1(0)$ & $2(0)$ & $5(0)$ & $18(5)$ & $50(10)$ \\
\hline Fainting & 654 & 20 & 3.1 & 19 & $1(0)$ & $1(0)$ & $2(0)$ & $-(0)$ & $-(1)$ \\
\hline Any HRI symptoms experienced at least once & 670 & 501 & 74.8 & - & - & - & - & - & - \\
\hline
\end{tabular}

${ }^{a}$ Numbers without parentheses indicate responses only from firefighters who experienced each symptom of HRI, while numbers in parentheses quantiles indicate answers from firefighters who have not experienced each symptom of HRI, which was coded as zero.

wear PPE. Frequency of HRI symptoms was also analysed with odds ratios where the variables were divided into two groups: 1) 5 and less than 5 times per year, 2) more than 5 times per year. The criterion dividing the two groups was determined based on the homogeneity in the answering frequency among adjacent numbers to the median. 95\% confidence interval and $p$-value were presented with odds ratios. In addition, Spearman's rank correlation test was used to analyse the relationship between the frequency of wearing PPE at rest and resting duration time with log transformation, and their correlation coefficients $(\rho)$ were presented. All $p$-values below 0.05 were considered to be statistically significant.

\section{Results}

\section{Frequency of HRI symptoms}

Seventy four point eight percent of firefighters out of 646 responded they have experienced at least one of the HRI symptoms asked. HRI symptom experienced by the largest number of firefighters was dizziness and more than half of firefighters experienced it (56.6\%) (Table 2). Headache $(48.8 \%)$, nausea $(47.5 \%)$, and sudden muscle cramps $(45.7 \%)$ were also reported by nearly half of respondents. Confusion was experienced by one third of respondents (33.2\%), 22.8\% of participants experienced vomiting, and 20 firefighters $(3.1 \%)$ fainted before in the work place (Table 2). Of the firefighters who have experienced HRI symptoms, three quarters of them reported that they experienced those all symptoms once per year or more than that. Moreover, half of them yielded 4 times of headache, 3 times of sudden muscle cramps, dizziness, confusion, and nausea, 2 times of vomiting, and once for fainting in a year. More emphasis is place on that there were great variances in the distribution so that $5 \%$ of respondents experienced more 30-50 times per year for headache, sudden muscle cramps, dizziness, confusion, and nausea and 95th percentile of vomiting was 18 times per year (Table 2). Those frequencies were 9-17 times the median. When all answers were used in the frequency analysis including 'no experience', results showed that $5 \%$ of all responders experienced approximately 20 times of dizziness, headache, nausea and muscle cramps, 12 times of confusion and 5 times of vomiting in a year. The results also showed that $2 \%$ of firefighters fainted at least once in a year (Table 2).

Among the 618 respondents who answered they have experienced HRI symptoms the vast majority of firefighters $(98.5 \%)$ answered they did not report it to the authority. The main reason was that they thought it was a natural phenomenon in the fire scenes (44.1\%). $16.9 \%$ of respondents answered that it was because other people do not usually report it, which was followed by the complexity in process $(12.3 \%)$ and troublesome (11.7\%). 9.9\% of participants explained the reason as little or no medical expense and $5.5 \%$ of firefighters were concerned about the potential of disadvantage for promotion. There were also other responses such as shame on their own physical capacity and moderate symptoms which can be rapidly recovered from based on their previous experiences.

\section{Duration time of rest and work period}

Duration time of rest and work period was separately presented according to the size of the fire accident in (Table 3). In small and medium size fire accidents, median 
Table 3. Korean firefighters' duration time of rest and work period in the fire scenes

\begin{tabular}{|c|c|c|c|c|c|c|c|c|c|c|}
\hline \multirow{4}{*}{$\begin{array}{c}\text { Phases } \\
\text { (Unit: min) }\end{array}$} & \multicolumn{10}{|c|}{ Size of fire accident ${ }^{\mathrm{a}}$} \\
\hline & \multicolumn{5}{|c|}{ Small-to-medium } & \multicolumn{5}{|c|}{ Conflagration $^{\mathrm{b}}$} \\
\hline & \multirow{2}{*}{$\mathrm{N}$} & \multicolumn{4}{|c|}{ Percentiles } & \multirow{2}{*}{$\mathrm{N}$} & \multicolumn{4}{|c|}{ Percentiles } \\
\hline & & 25 th & 50 th & 75 th & 95th & & 25 th & 50 th & 75 th & 95th \\
\hline \multicolumn{11}{|l|}{ Single rest period } \\
\hline Average & 599 & 7 & 10 & 15 & 30 & 585 & $10(1.4)$ & $15(1.5)$ & $21(1.4)$ & $45(1.5)$ \\
\hline Minimum & 626 & 5 & 5 & 10 & 20 & 614 & $5(1.0)$ & $10(2.0)$ & $15(1.5)$ & $30(1.5)$ \\
\hline Maximum & 625 & 10 & 20 & 29.5 & 50 & 614 & $15(1.5)$ & $20(1.0)$ & $30(1.0)$ & $60(1.2)$ \\
\hline \multicolumn{11}{|l|}{ Single work period } \\
\hline Average & 611 & 30 & 40 & 60 & 120 & 590 & $40(1.3)$ & $70(1.8)$ & $120(2.0)$ & $274.5(2.3)$ \\
\hline Minimum & 644 & 10 & 20 & 30 & 60 & 621 & $30(3.0)$ & $40(2.0)$ & $60(2.0)$ & $180(3.0)$ \\
\hline Maximum & 643 & 50 & 60 & 120 & 300 & 620 & $60(1.2)$ & $120(2.0)$ & $240(2.0)$ & $500(1.7)$ \\
\hline \multicolumn{11}{|l|}{ Total work period } \\
\hline Average & 579 & 50 & 65 & 120 & 240 & 560 & $88.5(1.8)$ & $141.5(2.2)$ & $240(2.0)$ & $628.5(2.6)$ \\
\hline Minimum & 608 & 20 & 30 & 60 & 115.5 & 589 & $50(2.5)$ & $70(2.3)$ & $120(2.0)$ & $300(2.6)$ \\
\hline Maximum & 607 & 90 & 140 & 240 & 720 & 589 & $140(1.6)$ & $260(1.9)$ & $480(1.0)$ & $1440(2.0)$ \\
\hline
\end{tabular}

aSize of fire accident is categorized in two groups; small-to-medium sized fire accident and conflagration.

${ }^{b}$ Values in parentheses indicate the ratio of values in conflagration compared with those in small-to-medium sized fire accident.

duration time of average rest period was $10 \mathrm{~min}$, but firefighters also reported $5 \mathrm{~min}$ as a minimum value. On the other hand, median duration time of a single work period was $40 \mathrm{~min}$ and $65 \mathrm{~min}$ for a total operation. Respondents also answered that they can work for $60 \mathrm{~min}$ in a single operation and $140 \mathrm{~min}$ for a total operation if the operation is prolonged, at most. However, there were great variances in distribution. 95th percentile of answers about the maximum duration time of a single work period was $300 \mathrm{~min}$, while $720 \mathrm{~min}$ for the total work period.

In case of conflagration, median duration time of working was almost double of those in smaller fire scenes, but resting time was not increased with an identical rate of working time. Average resting time in median was $15 \mathrm{~min}$, which was 1.5 times longer than the time of the smaller scenes. In particular, median maximal duration of rest period was $20 \mathrm{~min}$, which was identical with the time in the smaller scenes.

\section{Frequency of removing PPE at rest}

Helmet and gloves were the most frequently removed PPE during rest periods. $83.6 \%$ and $85.2 \%$ of respondents stated that they always or usually take off the helmet and protective gloves, respectively (Fig. 1). Sixty one point two percent of respondents responded that they always/ usually remove their hoods at rest. Although helmet, hood, and gloves were relatively frequently doffed during rest, there were firefighters who never removed these PPE even when they were resting (3.2\%, helmet; $5.6 \%$, hood;
$2.1 \%$, helmet). Regarding the bunker jacket, $28.7 \%$ of respondents answered that they always or usually take it off, $61.4 \%$ answered they usually just open it, and 9.8\% answered they neither open it nor take it off. On the other hand, in contrast of helmets, hoods and gloves, a majority of firefighters reported they do not take off bunker pants and boots or hardly take them off $(93.8 \%$, bunker pants; $88.9 \%$, boots).

\section{Relationship between HRI symptoms and resting/working time}

Frequency of HRI symptoms (dizziness, headache, nausea, muscle cramps, and confusion) showed no significant correlation with duration time of resting, but significant correlation with working hours was found (Table 4). In particular, average and maximum values of the single work period presented significant correlation coefficients with all reported HRI symptoms $(p<0.05)$. Of 5 HRI symptoms, muscle cramps and confusion showed significant correlation with all variables related to the work period $(p<0.05)$. They were weak correlations ranging from 0.105 to 0.263 , but they were statistically significant.

\section{Odds ratio of HRI symptoms according to wearing PPE}

Presences of HRI symptoms were statistically significantly greater in firefighters who usually wear PPE when resting versus firefighters who always/usually remove PPE: dizziness (gloves: OR 1.95, 95\% CI [1.23-3.09]); nausea (helmet: OR 2.01, 95\% CI [1.31-3.07]); vomiting 


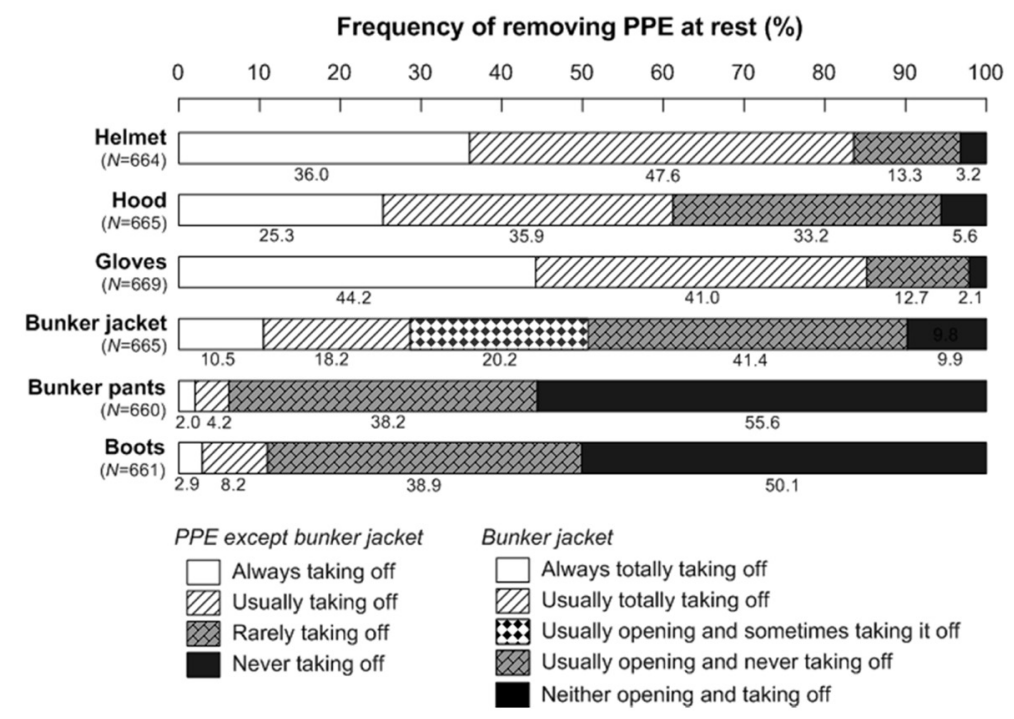

Fig. 1. Frequency of removing firefighters' personal protective equipment (PPE) during rest period.

(helmet: OR 2.16, 95\% CI [1.38-3.37]; gloves: OR 1.64, 95\% CI [1.02-2.64]); confusion (helmet: OR 2.07, 95\% CI [1.36-3.15]; gloves: OR 2.41, 95\% CI [1.02-2.64]); sudden muscle cramps (helmet: OR 1.67, 95\% CI [1.10-2.54]) (Table 5). Categorized experience frequencies also showed statistically significant increases when compared with wearing frequency of PPE during rest: dizziness (helmet: OR 2.62, 95\% CI [1.53-4.49]); nausea (helmet: OR 2.02, 95\% CI [1.14-3.57]; hood: OR 1.85, 95\% CI [1.12-3.05]; gloves: OR 1.90, 95\% CI [1.03-3.50]; boots: OR 2.44 [0.98-6.04]); confusion (gloves: OR 2.09, 95\% CI [1.074.08]; bunker jacket: OR 2.26, 95\% CI [1.06-4.84]).

\section{Resting duration time and removing PPE}

Firefighters, who had longer resting time more frequently removed their PPE, except the helmet and gloves: a hood $(r=0.158, p<0.001)$; a bunker jacket $(r=0.200$, $p<0.001)$; bunker pants $(r=0.136, p=0.001)$; boots $(r=0.182$, $p<0.001)$. Correlation coefficients between removing gloves and the resting time was $0.080(p=0.051)$.

\section{Discussion}

This study showed that firefighters' working hours and PPE worn during rest are related to the occurrence and the frequency of HRI symptoms. Furthermore, we documented the actual frequency of HRI symptoms in the line of duty by self-reporting firefighters' experience, which showed a wide variability between individuals. Many recent studies have investigated the effect of working conditions and cooling methods on physiological burden in experimental settings. A number of survey studies have explored the physical, chemical, and psychological factors including job stress to explain firefighters' comprehensive injuries. However, there has been a lack of empirical evidence about firefighters' HRI and its association with the working characteristics. This study specifically focused on speculating actual incidence rates of firefighters' HRI and its aggravating factors in the line of duty with nationwide survey data.

\section{Frequency and danger of HRI in the line of duty}

At first, this study revealed that there was great variability in experience frequency of HRI symptoms, and it is occurring more frequently than previous studies reported ${ }^{10}$. From the results of this study, $74.8 \%$ of firefighters experienced at least one of the above HRI symptoms throughout their career in the line of duty. Also, approximately $50 \%$ of respondents who reported the experience of HRI symptoms answered that they experienced dizziness, headache and nausea 3-4 times per year and more (Table 2). The distribution had a long tail toward the direction of high frequency and the upper $5 \%$ of respondents reported 9-16 times greater than the median frequency for the HRI symptoms (Table 2). With an assumption that the number of cases of nausea is equivalent with the number of cases of HRI, when extrapolated to the total population of firefighters in Korea who have charge of suppressing and rescuing work is approximately $60 \%$ of 40,406 except special employment for emergency medical services and 
Table 4. Correlation between experience frequency of heat-related illness (HRI) symptoms and resting/working hours

\begin{tabular}{|c|c|c|c|c|c|c|c|c|c|c|}
\hline \multirow{3}{*}{ Phases } & \multicolumn{10}{|c|}{ Experience frequency of HRI symptoms } \\
\hline & \multicolumn{2}{|c|}{ Dizziness } & \multicolumn{2}{|c|}{ Headache } & \multicolumn{2}{|c|}{ Nausea } & \multicolumn{2}{|c|}{ Muscle cramps } & \multicolumn{2}{|c|}{ Confusion } \\
\hline & $r$ & $p$ & $r$ & $p$ & $r$ & $p$ & $r$ & $p$ & $r$ & $p$ \\
\hline \multicolumn{11}{|c|}{ Single rest period } \\
\hline Average & N.S. & & N.S. & & N.S. & & N.S. & & N.S. & \\
\hline Minimum & N.S. & & N.S. & & N.S. & & N.S. & & N.S. & \\
\hline Maximum & N.S. & & N.S. & & N.S. & & N.S. & & N.S. & \\
\hline \multicolumn{11}{|l|}{ Work period } \\
\hline \multicolumn{11}{|c|}{ Single work period } \\
\hline Average & $0.154 * *$ & 0.004 & $0.210^{* *}$ & $<0.001$ & $0.228^{* *}$ & $<0.001$ & $0.223 * *$ & $<0.001$ & $0.225^{* *}$ & 0.001 \\
\hline Minimum & N.S & & $0.153^{* *}$ & 0.007 & $0.132 *$ & 0.020 & $0.201 * *$ & $<0.001$ & $0.263^{* *}$ & $<0.001$ \\
\hline Maximum & $0.105^{*}$ & 0.045 & $0.163 * *$ & 0.004 & $0.128^{*}$ & 0.025 & $0.196 * *$ & 0.001 & $0.182 * *$ & 0.008 \\
\hline \multicolumn{11}{|c|}{ Total work period } \\
\hline Average & N.S. & & $0.235 * *$ & $<0.001$ & $0.154 * *$ & 0.009 & $0.148 *$ & 0.014 & $0.227 * *$ & 0.001 \\
\hline Minimum & N.S. & & N.S. & & N.S. & & $0.171 * *$ & 0.004 & $0.163 *$ & 0.018 \\
\hline Maximum & $0.126^{*}$ & 0.017 & $0.199 * *$ & 0.001 & N.S. & & $0.158 * *$ & 0.007 & $0.168^{*}$ & 0.007 \\
\hline
\end{tabular}

Log transformation was done before correlation test with all data in symptoms of heat-related illness and the duration time of rest and work period. Pearson correlation test was used.

N.S.: not significant.

Table 5. Odds ratio of symptoms of heat-related illness (HRI) according to the frequency of taking off PPE

\begin{tabular}{|c|c|c|c|c|c|c|}
\hline \multirow[t]{2}{*}{ HRI symptoms/Wearing PPE } & \multicolumn{3}{|c|}{ Presence of HRI symptoms } & \multicolumn{3}{|c|}{ 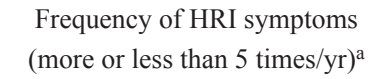 } \\
\hline & OR & CI $95 \%$ & $p$ & OR & CI 95\% & $p$ \\
\hline \multicolumn{7}{|l|}{ Dizziness } \\
\hline Wearing helmet at rest & 1.48 & $0.97-2.27$ & 0.070 & 2.62 & $1.53-4.49$ & $<0.001$ \\
\hline Wearing gloves at rest & 1.95 & $1.23-3.09$ & 0.004 & 1.51 & $0.87-2.60$ & 0.142 \\
\hline \multicolumn{7}{|l|}{ Nausea } \\
\hline Wearing helmet at rest & 2.01 & $1.31-3.07$ & 0.001 & 2.02 & $1.14-3.57$ & 0.014 \\
\hline Wearing hood at rest & 1.06 & $0.77-1.45$ & 0.727 & 1.85 & $1.12-3.05$ & 0.015 \\
\hline Wearing gloves at rest & 1.51 & $0.98-2.32$ & 0.062 & 1.90 & $1.03-3.50$ & 0.037 \\
\hline Wearing boots at rest & 0.69 & $0.42-1.13$ & 0.142 & 2.44 & $0.98-6.04$ & 0.048 \\
\hline \multicolumn{7}{|l|}{ Sudden muscle cramps } \\
\hline Wearing helmet at rest & 1.67 & $1.10-2.54$ & 0.015 & 1.80 & $0.99-3.26$ & 0.052 \\
\hline \multicolumn{7}{|l|}{ Confusion } \\
\hline Wearing helmet at rest & 2.07 & $1.36-3.15$ & 0.001 & 1.90 & $0.97-3.74$ & 0.060 \\
\hline Wearing gloves at rest & 2.41 & $1.56-3.71$ & $<0.001$ & 2.09 & $1.07-4.08$ & 0.030 \\
\hline Wearing bunker jacket at rest & 1.14 & $0.79-1.64$ & 0.478 & 2.26 & $1.06-4.84$ & 0.033 \\
\hline \multicolumn{7}{|l|}{ Vomiting } \\
\hline Wearing helmet at rest & 2.16 & $1.38-3.37$ & 0.001 & 1.43 & $0.56-3.64$ & 0.453 \\
\hline Wearing gloves at rest & 1.64 & $1.02-2.64$ & 0.040 & 2.47 & $0.97-6.30$ & 0.053 \\
\hline
\end{tabular}

${ }^{a}$ Experience frequency of symptoms of heat-related illness was categorized in two groups: more than 5 times per year (5 was not included) and less than 5 times (5 was included).

Frequency of wearing PPE during rest period was categorized in two groups: group of never/rarely taking off PPE and another group of always/usually taking off.

HRI: heat-related illness; OR: odds ratio; CI 95\%: 95\% confidence interval.

administrative personnel (MPSS 2015), it can be calculated that approximately 6,000 firefighters suffer from HRI
3 times or more in a year and 1,212 firefighters suffer HRI 20 times or more in a year. 
This calculation is not even close with previously reported numbers of HRI among firefighters ${ }^{10)}$, although little information about firefighters' HRI is available to precisely compare it with previous studies. Indeed, those studies and reports mostly dealt with the number of fatalities without consideration of mild HRI. Exceptionally, Bonauto and colleagues analysed occupational HRI cases in various forms using data from compensation insurance claims in Washington State for $11 \mathrm{yr}$ from 1995 to $2005^{10)}$. According to the results, firefighter was the second highest ranked occupation for the frequency of HRI claims by showing 39 claims (8.2\%) among the 480 HRI claims. Nevertheless, it is incomparably lower than the current results. Possibly, different methods used to collect HRI cases contributed it. Mild HRI symptoms such as headache and dizziness can also be simply regarded as overexertion and they can be recovered with appropriate recovery even without medical treatment. The authors also expressed concerns about unreported or unrecognized cases of HRI, since their HRI incidence case rate was far less than the incidence case rate of military recruits reported by Kark et $a l^{20)} ; 80.8$ claims per 100,000 persons per year engaged in fire protection ${ }^{10)}$ versus 2,686 cases per 100,000 persons per year occurred in US Marine recruits during $12 \mathrm{wk}$ basic training from 1982 to $1991^{20)}$. On the other hand, in the current study, the frequency of HRI was even much greater than the expected frequency from the cases in the military training ${ }^{20)}$. The main difference was the occupation (firefighters versus marine recruits) and its environmental settings (fire scene versus military training). Probably, different psychological motivation and responsibility might affect this difference, which can also be connected to the endurance level for managing heat stress. Additionally, different criteria in collecting HRI cases could be regarded as another main reason. The current study utilized selfreported frequency of previously identified HRI symptoms from dizziness to fainting to count expansive HRI cases which have not been recognized or not reported, which was also used by Fleischer et al. ${ }^{21)}$ about HRI cases in farm-workers, because of the difficulty to clinically diagnosis HRI and the passive attitude of subjects for the medical care. Meteorological characteristics on the summer in Korea could also be involved in the outstanding frequency of HRI symptoms. The average value of the daily highest temperature in Seoul in August 2016 was $32.63 \pm 4.08^{\circ} \mathrm{C}$ with $64 \pm 7 \%$ of relative humidity.

The current study also found that the majority of firefighters did not usually report their experiences of HRI symptoms $(98.5 \%)$ to the authority. The few cases of death caused by heat strain sometimes reported officially do not fully describe the risk of heat strain, as it does not reflect the other unrecorded HRI cases. Heat exhaustion is postulated to have the function of alarming the person about increased body core temperature by a central mechanism that protects the body from severe damage ${ }^{3)}$. Early recognition of the HRI symptoms and appropriate treatment can make them rest to cool down their body. Also, it is not only the problem of fatalities, but about the performance and ability to safely complete their duty in the fire scene because heat strain can negatively influence the accuracy of performance ${ }^{11)}$, which could be related to other accidents and injuries.

\section{Rest and work time and its association with HRI}

Due to the limited capacity of SCBA, firefighting operation is usually divided by intervals for changing SCBA or recharging it. Firefighters' single work time tends to be determined by air capacity of SCBA, but there are some cases of firefighters who do not wear SCBA in wildland firefighting or the operation of removing smouldering fire. Also, increased capacity of SCBA can allow firefighters to work much longer duration with increased concerns about heat strain. In this study, the median duration of a single firefighting episode was $40 \mathrm{~min}$ in the small-to-medium sized fires, whereas $70 \mathrm{~min}$ in the conflagration. $40 \mathrm{~min}$ is enough to increase firefighters' rectal temperature up to $39^{\circ} \mathrm{C}$ when high energy yield in the high ambient temperature is involved ${ }^{5)}$.

In addition, duration of work time is highly variable from short structural fires to long shifts over a day in wildland fires ${ }^{22}$, which means simply average or median work time cannot fully describe their working pattern. This study investigated the range of work time by asking their minimum, average, and maximum work time, separately. Working hours in the conflagration was additionally separately asked to segregate the cases occasionally occurring with the other cases firefighters are usually exposed to. The results showed great variability in working hours between and within individuals. In small-to-medium size fire scenes, the median value of average total work time was $65 \mathrm{~min}$, but the 95 th percentile was nearly 4 times of that $(240 \mathrm{~min})$, which indicates $5 \%$ of firefighters usually work for $4 \mathrm{~h}$ in the fire scene. In conflagration, $5 \%$ of firefighters answered that firefighting can be extended $24 \mathrm{~h}$ at maximum, whereas $1 \%$ of firefighters answered $3 \mathrm{~d}$. Such differences might be associated with not only the kind of firefighting (structural firefighting versus wildland firefighting), but also insufficiency in the number 
of personnel. In the previous interview for correcting this questionnaire, firefighters in rural areas stated that they are forced to work with a small number of firefighters because of lack of personnel. It also cannot guarantee enough rest time because they do not have extra firefighters to shift. These studies showed statistically significant correlations between this total work time and the occurrence of HRI symptoms, which implies that there is a lack of rehabilitation so that firefighters' physiological strain tends to be continuously accumulated.

Regarding rest time, it was documented that firefighters usually rest for $10 \mathrm{~min}$, but $25 \%$ of respondent reported less than $7 \mathrm{~min}$. NFPA 1584 recommends to rest for 10 to $20 \mathrm{~min}$ at least, but current resting hours of Korean firefighters do not fully meet this recommendation. According to Horn et $a l .{ }^{23)}$, even though firefighters start to take a rest, their rectal temperature continued to increase for $7 \mathrm{~min}$ after completion of previous activity $\left(0.020^{\circ} \mathrm{C} / \mathrm{min}\right)$. The current results did not present significant correlations between rest time and occurrence of HRI symptoms (Table 3), which rather emphasize the quality of rest, that is the efficiency in rehabilitation.

\section{Effect of removing PPE during rest}

Removing PPE is the simplest and the easiest cooling method. The results revealed that there were significant effects of wearing PPE during rest on occurrence of HRI symptoms (Table 4). Especially, firefighting helmets and gloves showed significant effects with presence or frequency of most of the HRI symptoms. The helmet showed significant effects on the presence of nausea, vomiting, confusion, and cramps with the frequency of dizziness, and nausea. Gloves showed significant effects on presence of dizziness, vomiting, and confusion with the frequency of nausea. The hood, bunker jacket, and boots showed significant effects on one of them, whereas the bunker pants showed no significant effect. However, this result should be carefully interpreted, because it did not indicate that the helmet and gloves have the most effective way to remove heat strain or that bunker pants do not have any effect on heat dissipation. This result is strongly related to the frequency of removing PPE during rest (Fig. 1). Because the helmet and gloves are the most frequently removed PPE during rest, it could be assumed that firefighters who do not remove the helmet or gloves have a much lower probability of removing other PPE, which could cause the relatively dramatic effect that removing the helmet and gloves has on heat strain.

On the other hand, the hood, bunker jacket, and boots also demonstrated the significant effect on partial HRI symptoms, which means reducing encapsulated body surface area is beneficial to decrease heat $\operatorname{strain}^{1,24)}$. However, this result cannot deny the effect that removing bunker pants has on HRI symptoms and on reducing heat strain, because the sample was highly biased to the population of rarely-to-never taking off PPE (93.8\%). Theoretically, removing the bunker jacket and pants is effective to cooling because it facilitates convective and evaporative heat loss in a larger portion of the body surface area. Guidelines for rehabilitation in NFPA 1584 urges firefighters to dress down by taking off their bunker coats, helmets, hoods, and opening the bunker pants as well ${ }^{15)}$. Furthermore, removing boots can effectively dissipate heat strain from the body, since the feet behave as remarkable radiators and evaporators, as well as the hands, with a great surface area to mass ratio (up to 3 times larger than that of the body) ${ }^{25}$.

However, this result also showed that such "dress down" methods are a matter of rest time. Wearing frequency of PPE showed negative correlation with rest time, except the helmet. NFPA 1584 recommends firefighters take a rest at least $10 \mathrm{~min}$ to $20 \mathrm{~min}$, but the median value for the average rest time in the small-to-medium fire was $10 \mathrm{~min}^{15)}$. Even $25 \%$ of respondents answered that they rest on average less than $7 \mathrm{~min}$, which is not enough time for removing PPE, except the helmet. In addition, don/ doff-friendly design is another issue here. Especially, when PPE is soaked by sweating or water from fire hoses, removing them gets far harder than before ${ }^{26)}$. Kim et al. ${ }^{26)}$ documented significantly extended time to don/doff wet gloves than dry ones, and 9 gloves among 13 made in US, Europe, Japan, and Korea showed problems of separated lining from the outer layer and coming off with the hands, which hindered firefighters successfully wearing them within a short time. The helmet is the only PPE which are not clearly interrupted by this problem. In the current survey, several firefighters gave the extra answer that they prefer a state of readiness, because they do not know when they have to re-enter fire scenes. Guaranteed sufficient rest time is needed along with improved PPE which can be easily donned/doffed even in the wet condition. Also, Additional cooling methods should be necessarily considered to refrain firefighters' heat strain accumulated throughout prolonged firefighting, in order to efficiently utilize the limited firefighters' rest time.

Although the crucial role of removing helmet and gloves to reduce heat strain can still be valid in the working place where neither sufficient resting time or rehabilitation strategies are given to firefighters. However, 
in another side of safety perspective, frequent removing PPE during rest periods can increase the risk of chemical exposure from not only smoke but also contaminated bunker gear by inhalation and through the skin ${ }^{17}$. Removing PPE is required to be promoted to firefighters, so that they recognize its critical contribution to their heat strain. At the same time, it should be noted that firefighters should take a measure to minimize the exposure to contaminants such as rehabbing away from the contaminated ensembles. Related to this issue, please refer following articles ${ }^{17,27)}$.

\section{Limitation}

This study postulated that symptoms experienced in the fire scene by firefighters such as dizziness, headache, nausea, confusion, vomiting and fainting are those of HRI, despite possibility of intervention from other diseases or problems. Hydration is another crucial rehabilitating component, but it was not included. In addition, potent confounding factors of HRI such as age, smoking, physical fitness, and BMI were not used to correct the statistical outcomes.

\section{Conclusion}

This study statistically showed that long durations of work as well as PPE worn during rest were related to the occurrence and the frequency of HRI symptoms as a result from a nationwide survey of Korean firefighters. Furthermore, we documented actual frequency of HRI symptoms in the line of duty, which was much greater than previous data. Huge variability in work or rest time and in experience frequency of HRI symptoms can be related to the type of firefighting and the insufficiency of personnel along with the possibility of individual vulnerability to heat. This study calls attention to firefighters' rehabilitation and provides empirical evidence on the need for systematic work and rest time and promotion of removing PPE during rest. Also, a priority on strategies that can be used in occupational environmental settings to resolve firefighters' excessive heat strain should be discussed in the current restricted environment. Ease of donning/doffing PPE should also be considered in designing them and the authority needs to consider it as one of the most crucial criteria in the decisions on purchasing PPE.

\section{References}

1) Lee JY, Kim S, Jang YJ, Baek YJ, Park J (2014) Component contribution of personal protective equipment to the alleviation of physiological strain in firefighters during work and recovery. Ergonomics 57, 1068-77. [Medline] [CrossRef]

2) Bouchama A, Knochel JP (2002) Heat stroke. N Engl J Med 346, 1978-88. [Medline] [CrossRef]

3) Becker JA, Stewart LK (2011) Heat-related illness. Am Fam Physician 83, 1325-30. [Medline]

4) Barr D, Gregson W, Reilly $T$ (2010) The thermal ergonomics of firefighting reviewed. Appl Ergon 41, 161-72. [Medline] [CrossRef]

5) McLellan TM, Selkirk GA (2006) The management of heat stress for the firefighter: a review of work conducted on behalf of the Toronto Fire Service. Ind Health 44, 414-26. [Medline] [CrossRef]

6) Lee JY, Park J, Park H, Coca A, Kim JH, Taylor NAS, Son SY, Tochihara Y (2015) What do firefighters desire from the next generation of personal protective equipment? Outcomes from an international survey. Ind Health 53, 434-44. [Medline] [CrossRef]

7) Young PM, Partington S, Wetherell MA, St Clair Gibson A, Partington E (2014) Stressors and coping strategies of U.K. firefighters during on-duty incidents. Stress Health 30, 366-76. [Medline] [CrossRef]

8) Walker A, Argus C, Driller M, Rattray B (2015) Repeat work bouts increase thermal strain for Australian firefighters working in the heat. Int J Occup Environ Health 21, 285-93. [Medline] [CrossRef]

9) The National Institute for Occupational Safety and Health. Wildland fire fighter dies from hyperthermia and exertional heat stroke while conducting mop-up operations-Texas. https://www.cdc.gov/niosh/fire/reports/face201117.html. Accessed April 2, 2018.

10) Bonauto D, Anderson R, Rauser E, Burke B (2007) Occupational heat illness in Washington State, 1995-2005. Am J Ind Med 50, 940-50. [Medline] [CrossRef]

11) Razmjou S, Kjellberg A (1992) Sustained attention and serial responding in heat: mental effort in the control of performance. Aviat Space Environ Med 63, 594-601. [Medline]

12) Razmjou S (1996) Mental workload in heat: toward a framework for analyses of stress states. Aviat Space Environ Med 67, 530-8. [Medline]

13) Kales SN, Soteriades ES, Christophi CA, Christiani DC (2007) Emergency duties and deaths from heart disease among firefighters in the United States. N Engl J Med 356, 1207-15. [Medline] [CrossRef]

14) Federal Emergency Management Agency. Emergency incident Rehabilitation. https://www.usfa.fema.gov/ downloads/pdf/publications/fa_314.pdf Accessed April 2, 2018.

15) NFPA 1584 (2015) Standard on the rehabilitation process for members during emergency operations and training exercises. National Fire Protection Association, Quincy.

16) Firefighters Health and Safety Regulations (2015) Ministry of Public Safety and Security (MPSS), Republic of Korea. 
17) Boorady LM, Barker J, Lee YA, Lin SH, Cho E, Ashdwon SP (2013) Exploration of firefighter turnout gear, Part I: Identifying male firefighter user needs. J Text Apparel Tech Manage 8, 1-13.

18) Fent KW, Eisenberg J, Snawder J, Sammons D, Pleil JD, Stiegel MA, Mueller C, Horn GP, Dalton J (2014) Systemic exposure to PAHs and benzene in firefighters suppressing controlled structure fires. Ann Occup Hyg 58, 830-45. [Medline]

19) Pryor RR, Roth RN, Suyama J, Hostler D (2015) Exertional heat illness: emerging concepts and advances in prehospital care. Prehosp Disaster Med 30, 297-305. [Medline] [CrossRef]

20) Kark JA, Burr PQ, Wenger CB, Gastaldo E, Gardner JW (1996) Exertional heat illness in Marine Corps recruit training. Aviat Space Environ Med 67, 354-60. [Medline]

21) Fleischer NL, Tiesman HM, Sumitani J, Mize T, Amarnath KK, Bayakly AR, Murphy MW (2013) Public health impact of heat-related illness among migrant farmworkers. Am J Prev Med 44, 199-206. [Medline] [CrossRef]

22) Raines J, Snow R, Petersen A, Harvey J, Nichols D, Aisbett B (2012) Pre-shift fluid intake: effect on physiology, work and drinking during emergency wildfire fighting. Appl
Ergon 43, 532-40. [Medline] [CrossRef]

23) Horn GP, Gutzmer S, Fahs CA, Petruzzello SJ, Goldstein E, Fahey GC, Fernhall B, Smith DL (2011) Physiological recovery from firefighting activities in rehabilitation and beyond. Prehosp Emerg Care 15, 214-25. [Medline] [CrossRef]

24) Kim S, Jang YJ, Baek YJ, Lee JY (2014) Influences of partial components in firefighters' personal protective equipment on subjective perception. Fashion Text 1, 1-14.

25) Taylor NA, Machado-Moreira CA, van den Heuvel AM, Caldwell JN (2014) Hands and feet: physiological insulators, radiators and evaporators. Eur J Appl Physiol 114, 2037-60. [Medline] [CrossRef]

26) Kim D, Lee I, Lee JY (2016) Mobility evaluation of popular firefighting protective gloves in domestic and foreign countries: don-doff test, dexterity test and torque test. J Korean Soc Cloth Text 40, 921-35. [CrossRef]

27) Fent KW, Horn GP, Kirk KM, Logan MB. Off-Gassing Contaminants from Firefighters' Personal Protective Equipment, September 2015. https://www.fireengineering. com/articles/print/volume-168/issue-9/features/off-gassingcontaminants-from-firefighters-personal-protectiveequipment.html. Accessed July 15, 2018. 